\title{
Genome-wide analysis of long non-coding RNAs in Catalpa bungei and their potential function in floral transition using high- throughput sequencing
}

\author{
Zhi Wang ${ }^{1}$, Tianqing Zhu' ${ }^{1}$, Wenjun Ma', Nan Wang ${ }^{1}$, Guanzheng $\mathrm{Qu}^{2}$, Shougong Zhang ${ }^{1}$ and Junhui Wang ${ }^{1 *}$ (D)
}

\begin{abstract}
Background: Long non-coding RNAs (IncRNAs) have crucial roles in various biological regulatory processes. However, the study of IncRNAs is limited in woody plants. Catalpa bungei is a valuable ornamental tree with a long cultivation history in China, and a deeper understanding of the floral transition mechanism in C. bungei would be interesting from both economic and scientific perspectives.

Results: In this study, we categorized C. bungei buds from early flowering (EF) and normal flowering (NF) varieties into three consecutive developmental stages. These buds were used to systematically study IncRNAs during floral transition using high-throughput sequencing to identify molecular regulatory networks. Quantitative real-time PCR was performed to study RNA expression changes in different stages. In total, 12,532 IncRNAs and 26,936 messenger RNAs (mRNAs) were detected. Moreover, 680 differentially expressed genes and 817 differentially expressed IncRNAs were detected during the initiation of floral transition. The results highlight the mRNAs and IncRNAs that may be involved in floral transition, as well as the many IncRNAs serving as microRNA precursors. We predicted the functions of IncRNAs by analysing the relationships between IncRNAs and mRNAs. Seven IncRNA-mRNA interaction pairs may participate in floral transition.
\end{abstract}

Conclusions: This study is the first to identify IncRNAs and their potential functions in floral transition, providing a starting point for detailed determination of the functions of IncRNAs in C. bungei.

Keywords: Long non-coding RNAs, Woody plant, Floral transition, RNA-sequencing, Catalpa bungei

\section{Background}

In the early 1990s, a new class of RNA was identified [1-3]; the RNAs in this class, known as non-coding RNAs (ncRNAs), are transcripts that lack protein-coding potential [4]. Based on product length, ncRNAs can be subdivided into two groups: small ncRNAs $(<200 \mathrm{nt})$, which are mainly microRNAs (miRNAs) and small RNAs (sRNAs), and long ncRNAs (lncRNAs; > $200 \mathrm{nt}$ ), which include long intronic ncRNAs and long intergenic ncRNAs [5]. IncRNAs can be divided into three forms

\footnotetext{
* Correspondence: wangjh808@sina.com; wangjh@caf.ac.cn

'State Key Laboratory of Tree Genetics and Breeding, Key Laboratory of Tree Breeding and Cultivation of State Forestry Administration, Research Institute of Forestry, Chinese Academy of Forestry, Haidian District, Dongxiaofu 1\#, Beijing 100091, People's Republic of China

Full list of author information is available at the end of the article
}

based on their effects: miRNA precursors, natural antisense transcripts (NATs), and lncRNAs that bind with miRNAs to sequester the regulatory roles of miRNAs on their target genes.

High-throughput genomic technologies have advanced our understanding of lncRNAs in the last decade. However, most studies on lncRNAs have concentrated on animals, and few studies have been performed in plants $[2,5]$. To date, IncRNAs have been studied in only some plant species, mostly vegetables or herbs, but also several woody plants [6-8].

In plants, lncRNAs are considered important in fertility [7], fruit ripening [9], DNA methylation [10, 11], flowering time [12, 13], and photomorphogenesis [14]. COLDAIR and COOLAIR are the most well studied

(c) The Author(s). 2018 Open Access This article is distributed under the terms of the Creative Commons Attribution 4.0 International License (http://creativecommons.org/licenses/by/4.0/) which permits unrestricted use, distribution, and reproduction in any medium, provided you give appropriate credit to the original author(s) and the source, provide a link to the Creative Commons license, and indicate if changes were made. The Creative Commons Public Domain Dedication waiver (http://creativecommons.org/publicdomain/zero/1.0/) applies to the data made available in this article, unless otherwise stated. 
lncRNAs in plants [12-16]; they participate in the repression of Flowering Locus C (FLC) during vernalization in Arabidopsis and are involved in flower transition [12]. However, few studies on lncRNAs related to floral transition in woody plants are available. Catalpa bungei (family: Bignoniaceae) is a valuable timber material also used in Chinese medicine. Furthermore, $C$. bungei is famous for its beautiful flowers and is an ancient ornamental woody plant widely distributed in the middle and western regions of China. C. bungei typically experiences its first floral transition in trees that are five years old or older. However, an early flowering $(E F)$ variety undergoes flower transition in the first year after planting. Here, we used this EF variety to study the lncRNAs expressed during the transition from vegetative growth to reproductive growth in C. bungei with plants provided by the Henan Provincial Department of Forestry (http://www.hnly.gov.cn).

The Illumina Hi-Seq sequencing platform was used to further investigate the genes and lncRNAs involved in floral transition. In the present study, a comprehensive analysis of lncRNAs and mRNAs from EF and normal flowering (NF) varieties during three development periods was performed. A total of 680 differentially expressed genes (DEGs) and 817 differentially expressed lncRNAs (DELs) were identified during the initiation of floral transition. Further analysis indicated that lncRNAs show several distinctions from mRNAs. For example, numerous lncRNAs are precursors to miRNAs and many lncRNAs regulate protein-coding genes' expression. This study provides fundamental information to aid future investigations of floral transition in C. bungei. This is the first study to identify and characterize the lncRNAs present during floral transition in woody plants.

\section{Methods}

\section{Plant materials}

C. bungei is a perennial tree that typically flowers after seven years. However, a natural EF variety of $C$. bungei that flowers after one year was found in Henan Province, China, and was used to create a new variety, "bairihua". From February 28 to March 31, 2016, we collected the first round of axillary buds of EF and NF varieties every one to two days. The samples used for RNA extraction were washed with distilled water, frozen immediately in liquid nitrogen, and stored at $-80^{\circ} \mathrm{C}$. Samples for histological analysis were fixed in formalin: glacial acetic acid: $70 \%$ ethanol (5:5:90 vol.; FAA) solution under a vacuum for at least $24 \mathrm{~h}$.

\section{Histological analysis}

After FAA fixation, the samples were dehydrated according to the methods of a previous study [17-19], processed with three changes of $100 \%$ paraffin at $63{ }^{\circ} \mathrm{C}$, and finally embedded. The paraffin-embedded material was cut into 10-mm-thick sections (RM2255 Fully Automated Rotary Microtome; Leica, Germany), and the sections were stained with Safranin O and fast green FCF (Sigma-Aldrich, USA) [17]. The slices were observed and photographed using a Nikon D3000 camera, a Leica M205 FA fluorescence stereo microscope, and a Leica DM 6000B fully automated upright microscope (Leica Microsystems GmbH, Wetzlar, Germany).

\section{Total RNA isolation, library construction, and Illumina transcriptome sequencing}

Total RNA was isolated using the Spectrum Plant Total RNA Kit (Sigma-Aldrich, USA) following the manufacturer's protocol. Total RNA quality was monitored by ultraviolet spectrophotometry (NanoDrop 8000 Spectrophotometer; Thermo Scientific, USA). First-stand complementary DNA (cDNA) was synthesized using the First Strand cDNA Synthesis Kit (TaKaRa, Japan) following the manufacturer's instructions.

The EF and NF buds were grouped into three developmental periods according to the histological analysis. Three replicates were included for each period. Libraries were constructed using the second-generation TruSeq Stranded RNA Kit (Illumina Inc., San Diego, USA) following the manufacturer's recommendations. In total, 18 cDNA libraries, which were sequenced using an Illumina Solexa sequencer, were constructed. To assess the quality of the RNA-sequencing (RNA-seq) data, each base in the reads was assigned a quality score $(\mathrm{Q})$ with a Phred-like algorithm using SOAPnuke software (http:// soap.genomics.org.cn/) [20].

\section{IncRNA identification}

Transcripts were assembled and merged using Cufflinks software according to the software instructions [21]. The initial assembled transcripts were compared to known $C$. bungei transcripts using Cuffcompare software [22-24]. High-quality assemblies with lengths $\geq 200$ bp were retrieved. Three prediction programmes, CPC (http:// www.mybiosoftware.com/cpc-0-9r2-assess-protein-coding-potential-transcripts.html), txCdspredict (http://hgdownload.soe.ucsc.edu/admin/jksrc.zip), and CNCI (https:// github.com/www-bioinfo-org/CNCI), were used to predict the protein-coding ability of the transcripts [2224]. Score thresholds were set to distinguish lncRNA from mRNA (CPC threshold: $\geq 0=$ mRNA, $<0=$ IncRNA; txCdspredict threshold: $\geq 500=$ mRNA, < $500=$ lncRNA; CNCI threshold: $\geq 0=$ mRNA, $<0=$ lncRNA) [22]. Transcripts that could be aligned in the protein database Pfam were predicted to be mRNA, while those that could not be aligned in Pfam were predicted to be IncRNA [25]. Transcripts reported as 
lncRNAs by at least three of the four above prediction methods were identified as IncRNAs.

\section{IncRNAs predicted to be miRNA targets}

DEL target genes were identified based on their trans-regulatory effects using sequence complementary analysis, as described previously. To explore whether lncRNAs function as miRNA decoys, the lncRNAs were submitted to the psRNATarget server (http://plantgrn.noble.org/ psRNATarget/) with an expectation value $<3$. IncRNAs containing no more than four mismatches and G/U pairs within the IncRNA and miRNA complementary regions were considered miRNA targets.

\section{Analysis of the positional relationship between IncRNAs and mRNAs}

lncRNAs regulate target genes via proximal (cis) or remote (trans) control. lncRNAs were identified as cis if they were located upstream of the mRNA or within $20 \mathrm{k}$ downstream. Beyond this range, trans lncRNAs did not rely on the locational relationship, and the binding energy had to be calculated. RNAplex software was used to analyse the binding energy of the lncRNAs and mRNAs. If the binding energy was $<30$, then the lncRNA was identified as trans and the mRNA adjacent to the IncRNA was screened as its target gene. Spearman and Pearson correlation coefficients were used to screen target genes, with eligibility as a target defined by a Spearman correlation coefficient $\geq 0.6$ and a Pearson correlation coefficient $\geq 0.6$.

\section{IncRNA identification as miRNA precursors}

To identify lncRNAs acting as precursors of known or novel miRNAs, lncRNAs were aligned with precursors of known miRNAs in the miRBase 21.0 database (http:// www.mirbase.org/) with the NCBI Basic Local Alignment Search Tool (BLAST; https://blast.ncbi.nlm.nih.gov/Blast. cgi) using the default parameters. IncRNAs homologous to miRNA precursors with $>90 \%$ coverage were defined as miRNA precursors.

\section{Expression analysis}

The expression levels of all transcripts, including lncRNAs and mRNAs, in the NF and EF buds were quantified as the fragments per kilobase of exon per million fragments mapped (FPKM) using the Cuffdiff programme from the Cufflinks package [24, 26-28]. The multiread and fragment bias correction methods embedded in Cufflinks were adopted to improve the accuracy of expression level estimations. DEGs were identified using the DESeq package with an adjusted $P$-value of 0.01 and a fold change of at least $1.2[28]$.
Analysis of IncRNAs near coding genes and prediction of IncRNA families

To better annotate and understand the functions of the predicted lncRNAs, the lncRNAs were classified into different families according to their evolutionary ancestor using the Rfam database (http://rfam.xfam.org/) and INFERNAL software (http://eddylab.org/infernal/) with the default parameters $[24,25,29]$.

\section{Function prediction and IncRNA and mRNA enrichment} All assembled transcripts were annotated using the publicly available Gene Ontology (GO; http://www.geneonto logy.org) protein database. Gene function enrichment was calculated based on a hypergeometric distribution. The phyper function in $\mathrm{R}$ was used to analyse the $P$-value for each function theme:

$$
\mathrm{P}=1-\sum_{i=0}^{m-1} \frac{\left(\begin{array}{c}
M \\
i
\end{array}\right)\left(\begin{array}{c}
N-M \\
n-i
\end{array}\right)}{\left(\begin{array}{c}
N \\
n
\end{array}\right)}
$$

Smaller $P$-values were associated with greater enrichment of the candidate genes in a given function theme (https:// en.wikipedia.org/wiki/Hypergeometric_distribution).

\section{Orthologue analysis of genes involved in floral transition in C. bungei}

Arabidopsis genes related to floral transition were obtained from previous studies [30-36]. The gene sequences were downloaded from The Arabidopsis Information Resource (http://www.arabidopsis.org/). The putative orthologue pairs from Arabidopsis were analysed using BLAST [28]. Based on the data obtained from BLAST, we identified the homologous genes of interest in C. bungei (Additional file 1).

\section{Quantitative real-time (qRT)-PCR and correlation analysis of expression trends}

Total RNA was extracted using an RNA reagent kit (RN38; Aidlab Biotechnology, Beijing, China) according to the manufacturer's protocol and were treated with RNase-free DNase I (Takara, Dalian, China) to remove genomic DNA contamination. The qRT-PCR analyses were conducted with a 7500 Real-Time PCR System (Applied Biosystems, CA, USA) using the SYBR Premix Ex Taq Kit (TaKaRa, Dalian, China) following the manufacturer's instructions. Relative expression levels were calculated using the $2^{-\Delta \Delta \mathrm{Ct}}$ method. Cbu-actin and U6 were amplified as an endogenous control [18]. All the primers are shown in Additional file 2. We test the correlation of expression (CEG) between lncRNAs/miRNAs and $\operatorname{lncRNAs} / \mathrm{mRNAs}$ by using the Pearson correlation 


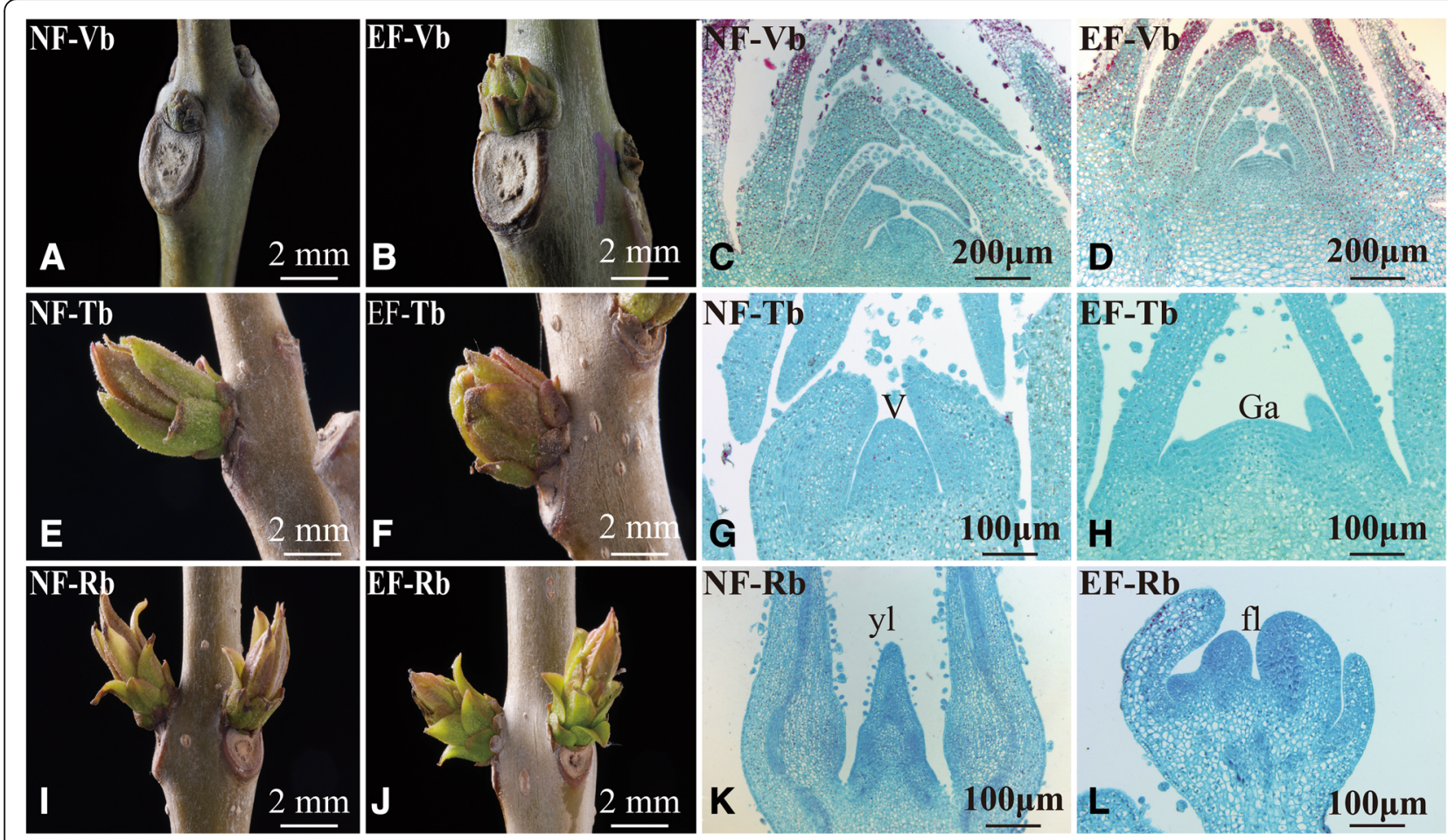

Fig. 1 External and internal morphologies of buds from early flowering (EF) and normal flowering (NF) C. bungei. a-d Images and sections of vegetative buds (Vbs) from NF and EF varieties. a Image of a Vb from the NF variety (NF-Vb). b Image of a Vb from the EF variety (EF-Vb). c Section of an NF-Vb. d Section of an EF-Vb. e-h Images and sections of transition buds (Tbs) from the NF and EF varieties. e Image of an NF-Tb. $\mathbf{f} I m a g e$ of an EF-Tb. $\mathbf{g}$ Section of an NF-Tb, note the bulged vegetative apex ( $)$. $\mathbf{h}$ Section of an EF-Tb, note the flat generative apex (Ga). $\mathbf{i}-\mathbf{I}$ Images and sections of reproductive buds (Rbs) from the NF and EF varieties. $\mathbf{i}$ Image of an NF-Rb. $\mathbf{j}$ Image of an EF-Rb. $\mathbf{k}$ Section of an NF-Rb, note the leaf primordium (Ip). I Section of an EF-Tb, note the flower primordium (fl)

coefficient. The Pearson correlation coefficient was calculated by $\operatorname{COR}()$ in $\mathrm{R}$ [37].

\section{Data access}

The stranded RNA-seq datasets are available in the Sequence Read Archive database in NCBI (accession number: SRP120718).

\section{Results}

Morphological analysis of EF and NF buds from C. bungei The major distinguishing characteristic of the EF variety is its one-year juvenile period; in contrast, the NF variety has a minimum juvenile period of five years. To study the external and internal morphologies of EF and NF buds in different growth phases, we collected buds from two-year-old EF and NF plants. Changes in bud appearance were recorded and paraffin sections were produced to observe the internal morphologies of the buds in the corresponding stages.

In the early stage, the buds from the EF and NF plants were vegetative buds (Vbs). Although the EF-Vbs were generally larger than the NF-Vbs (Fig. 1a, b), no differences were observed in the sections (Fig. 1c, d). With continued plant growth, the NF buds generally became

Table 1 Statistical data of the RNA-SEQ

\begin{tabular}{|c|c|c|c|c|c|c|}
\hline & \multicolumn{3}{|l|}{ NF } & \multicolumn{3}{|l|}{$\mathrm{EF}$} \\
\hline & $\mathrm{Vb}$ & $\mathrm{Tb}$ & $\mathrm{Rb}$ & $\overline{\mathrm{Vb}}$ & $\mathrm{Tb}$ & $\mathrm{Rb}$ \\
\hline Raw reads & $1,317,350,57.3$ & $131,393,398$ & 1.313 .956 .05 .3 & $131,925,484$ & 1.317 .243 .187 & $1,315,289,37.3$ \\
\hline Clean read & $88,179,437.33$ & $88,524,307$ & $89,997,175.30$ & $90,551,314$ & $83,876,034.60$ & $87,239,646$ \\
\hline Total Mapping Ratio(IncRNAs) & $75.91 \%$ & $76.00 \%$ & $76.25 \%$ & $78.19 \%$ & $75.51 \%$ & $76.66 \%$ \\
\hline Total Mapping Ratio (mRNAs) & $73.93 \%$ & $74.24 \%$ & $74.98 \%$ & $74.51 \%$ & $73.29 \%$ & $75.52 \%$ \\
\hline Uniquely Mapping Ratio (IncRNAs) & $71.90 \%$ & $71.99 \%$ & $72.26 \%$ & $74.23 \%$ & $71.66 \%$ & $72.55 \%$ \\
\hline Uniquely Mapping Ratio (mRNAs) & $26.07 \%$ & $25.76 \%$ & $25.02 \%$ & $25.49 \%$ & $26.71 \%$ & $24.48 \%$ \\
\hline
\end{tabular}



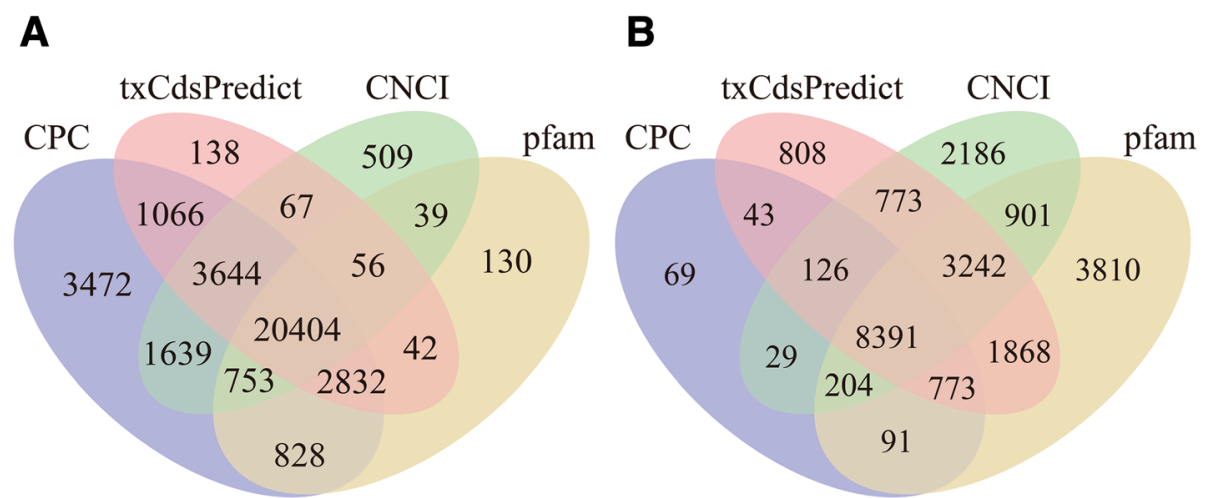

Fig. 2 Venn plots showing mRNA and IncRNA predictions. a Predicted coding genes. b Predicted non-coding genes. CPC1, txCdspredict, CNCI2, and Pfam3 were used to predict the mRNAs and IncRNAs. The parameters of the three programmes used for prediction are as follows: CPC threshold, $\geq 0=$ mRNA,$<0=\operatorname{IncRNA}$; txCdspredict threshold, $\geq 500=$ mRNA, $<500=\operatorname{IncRNA}$; CNCl threshold $, \geq 0=m R N A,<0=\operatorname{IncRNA}$. Pfam is a protein-coding sequence database. Transcripts were confirmed as IncRNAs or mRNAs through prediction by at least three of the four methods

longer and thinner than the swollen EF buds (Fig. 1e, f). Conversely, the internal morphologies of the buds in this stage differed substantially. The apex in the NF buds bulged, indicating that they were still in the vegetative growth stage (Fig. 1g). In comparison, a flattened generative apex, which marked the floral transition, developed in the EF buds (Fig. 1h), which were considered transition buds (Tbs).

The morphological differences between the EF and NF buds became more evident over time. The NF buds remained in the vegetative stage, with elongated leaf primordia (Fig. 1i, k), whereas the EF buds developed into reproductive buds (Rbs) that had completed the transformation from vegetative growth to reproductive growth and had begun differentiation into the flower primordia (fl) (Fig. 1j, l). Notably, even though floral transition was never observed in the NF buds, the NF buds collected in the period corresponding to floral transition are henceforth called Tbs or Rbs for convenience.

Figure 1 shows the external and internal morphologies of the EF and NF buds in the three growth phases. The appearance of the generative apex marked the occurrence of floral transition, whereas the initiation of the flower primordia marked the completion of floral transition. The results indicated that the early vegetative-reproductive transition only occurred in the EF buds, not the NF buds. The EF and NF samples were divided into six groups according to these results for the following experiments.

\section{High-throughput sequencing}

We performed 150-bp paired-end sequencing on raw reads using the Illumina HiSeq 4000 platform by BGI. The total initial reads were processed with in-house Perl scripts. The reads with more than $0.02 \%$ unknown bases, the reads with more than $0.68 \%$ adapter bases and the reads with more than $0.17 \%$ low-quality bases were removed, yielding 13G high-quality (Phred-like Q20, Q30, and GC content) raw reads [20,38,39] (Additional file 3). The reads with more than $0.02 \%$ rRNA and the reads with more than $28.74 \%$ duplications were removed from the $13 \mathrm{G}$ raw reads, yielding $9 \mathrm{G}$ clean reads (Table 1). All the following analyses were based on the $13 \mathrm{G}$ raw reads and $9 \mathrm{G}$ clean reads (Additional file 4). From our data, more than $70 \%$ of the IncRNAs and mRNAs were mapped to the $C$. bungei genome [40, 41]. In total, 12,532 lncRNAs and 26,936 mRNAs were obtained (Fig. 2), with $82.4 \%$ of the lncRNA transcripts distributed within the length range of $0-1000 \mathrm{nt}$, and $74.1 \%$ of the mRNA transcripts were shorter than $2500 \mathrm{nt}$. Most of

Table 2 List of the IncRNAs transcripts predicted to be miRNA precursors

\begin{tabular}{lll}
\hline LncRNA ID & miRNA ID & LncRNA length/bp \\
\hline ICI|Mguttatus_Migut.J01166.1_dup1 & ath-MIR414 & 323 \\
IcI|Mguttatus_Migut.I00370.1_dup1 & gga-MIR466 & 788 \\
ICI|Mguttatus_Migut.M01324.1_dup1 & vvi-MIR171d & 1166 \\
IcI|Mguttatus_Migut.M01324.1_dup1 & mes-MIR171c & 1166 \\
Ic||Mguttatus_Migut.M01324.1_dup1 & vvi-MIR171j & 1166 \\
LXLOC_019956 & aqc-MIR156b & 591 \\
LXLOC_023659 & peu-MIR2916 & 1461 \\
LXLOC_024876 & ssl-MIR171a & 1489 \\
LXLOC_019956 & mes-MIR156h & 591 \\
LXLOC_019956 & mes-MIR156i & 591 \\
LXLOC_019956 & mes-MIR156j & 591 \\
LXLOC_008074 & mes-MIR160d & 4648 \\
LXLOC_008074 & mes-MIR160h & 4648 \\
LXLOC_008074 & cpa-MIR160C & 4648 \\
LXLOC_008074 & cpa-MIR160f & 4648 \\
\hline
\end{tabular}



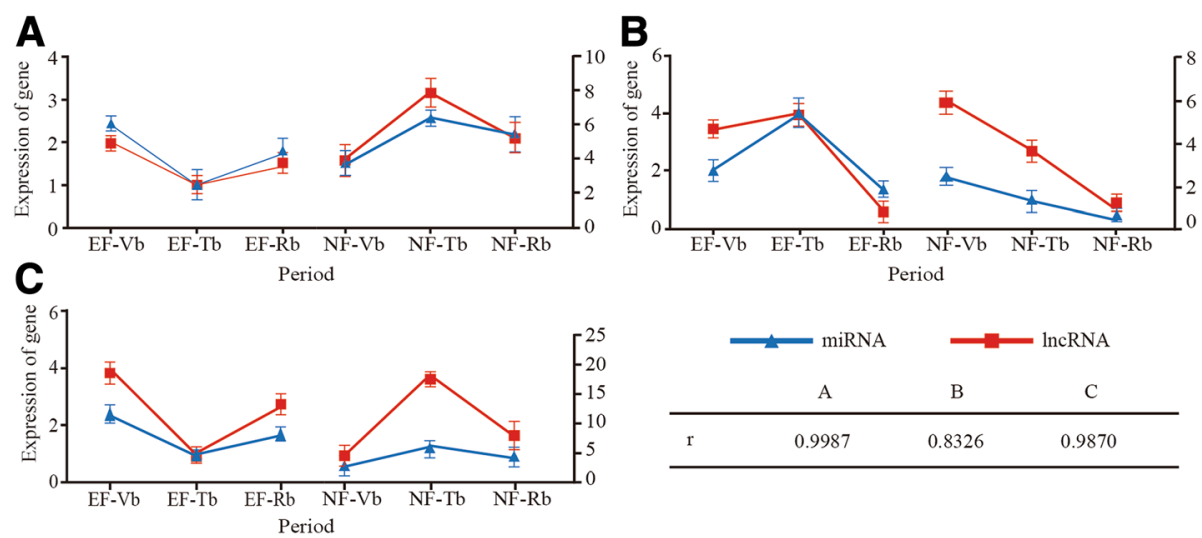

Fig. 3 The correlation coefficients of expression of IncRNAs and miRNAs between the EF and NF varieties during three developmental periods. a The expression of LXLOC.019956-miR156 between the EF and NF varieties during the three developmental periods. b The expression of LXLOC.024876miR160 between the EF and NF varieties during the three developmental periods. $\mathbf{c}$ The expression of LXLOC.008074-miR171 between the EF and NF varieties during the three developmental periods. The red line with a square represents the expression of IncRNA, and the blue line with a triangle represents the expression of miRNA. $r$ is the correlation coefficient of expression of the three "co-expression" pairs

the lncRNAs (81.4\%) contained one to two exons, whereas most of the mRNAs (59.2\%) contained three to more than ten (11.2\%) exons (Additional file 5) [9].

\section{Prediction of IncRNA transcripts as miRNA precursors} lncRNAs that matched miRNA precursors with more than $90 \%$ similarity were selected as potential precursors of the corresponding miRNAs, and seven lncRNAs were identified. Of these, three were known lncRNAs and four were unknown lncRNAs, including precursors for six
miRNA families (miR414, miR466, miR171, miR156, miR2916, and miR160) (Table 2, Additional file 6). To further study the correlations between lncRNAs and miRNAs, we analyzed their CEGs in the EF and NF samples. In-depth research was performed on three of the six miRNA families: miR156, miR160, and miR171. The results showed that lncRNAs and miRNAs have similar positive correlations with $r>0.8$ (Fig. 3), implying that IncRNAs may be able to indirectly regulate the floral transition as miRNA precursors.

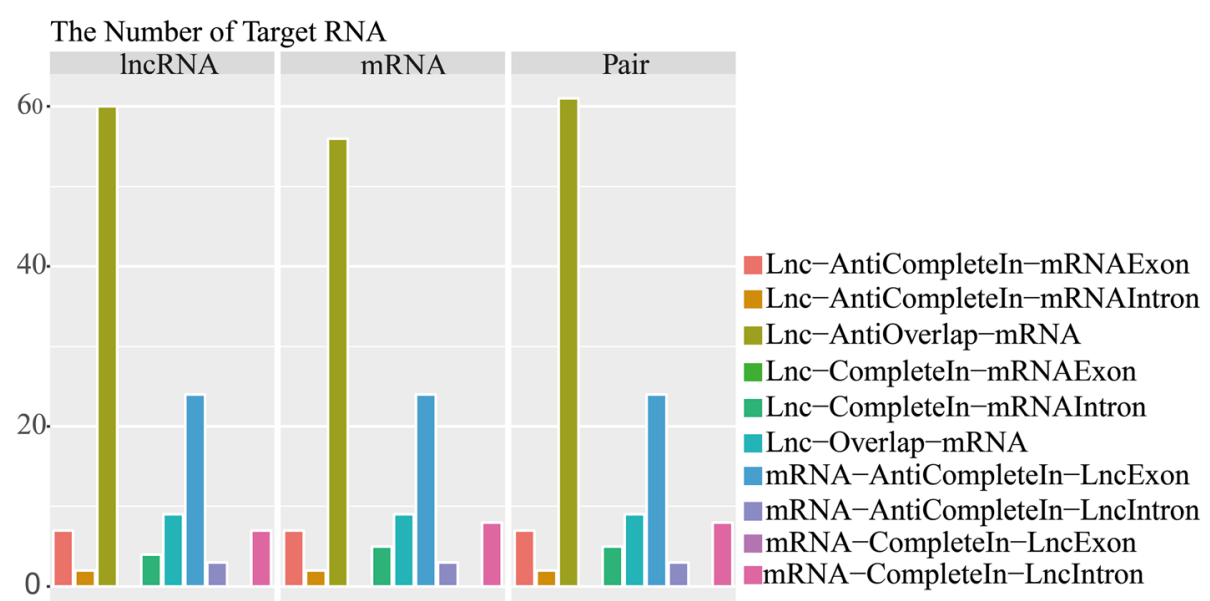

Fig. 4 Column plot showing the number of IncRNA and mRNA interaction pairs in different types. Lnc-AntiCompleteln-mRNAExon: the IncRNA and mRNA are in different chains and the IncRNA is completely within the exon of the mRNA; Lnc-AntiCompleteln-mRNAIntron: the IncRNA and mRNA are in different chains, and the IncRNA is completely within the intron of the mRNA; Lnc-Antioverlap-mRNA: the IncRNA and mRNA are in different chains but partially overlap; Lnc-Completeln-mRNAExon: the IncRNA is in the same chain as the mRNA, and the IncRNA is completely within the exon of the mRNA; Lnc-Completeln-mRNAIntron: the IncRNA is in the same chain as the mRNA, and the IncRNA is completely within the intron of the mRNA; Lnc-Overlap-mRNA: the IncRNA is in the same chain as the mRNA, and the IncRNA and mRNA partially overlap; mRNA-AntiCompletelnLncExon: the IncRNA and mRNA are in different chains, and the mRNA is completely within the exon of the IncRNA; mRNA-AntiCompleteln-Lnclntron: the IncRNA and mRNA are in different chains, and the mRNA is completely within the intron of the IncRNA; mRNA-Completeln-LncExon: the IncRNA is in the same chain as the mRNA, and the mRNA is completely within the exon of the IncRNA; mRNA-Completeln-LncIntron: the IncRNA is in the same chain as the mRNA, and the mRNA is completely within the intron of the IncRNA 
Table 3 The IncRNAs-mRNA pairs involving in floral transition

\begin{tabular}{lllll}
\hline LnCRNA & mRNA & IncRNA length/bp & mRNA length/bp & Annotation \\
\hline LXLOC_006261 & Cbu.gene.20428 & 2519 & 975 & NAC homologous gene \\
LXLOC_019079 & Cbu.gene.1092 & 1598 & 1657 & TRY homologous gene \\
LXLOC_019953 & Cbu.gene.190 & 943 & 1872 & NF-YC homologous gene \\
LXLOC_017817 & Cbu.gene.24144 & 1392 & 641 & SOC1 homologous gene \\
LXLOC_030659 & Cbu.gene.7250 & 766 & 922 & SOC1 homologous gene \\
LXLOC_025313 & Cbu.gene.18564 & 694 & 1300 & AP2 homologous gene \\
LXLOC_004449 & Cbu.gene.27201 & 1877 & 738 & AP2 homologous gene \\
\hline
\end{tabular}

\section{IncRNA target prediction}

In total, 119 lncRNA-mRNA pairs were identified using this method, including 61 Lnc-Antioverlap-mRNA pairs. Interestingly, these 61 pairs included 60 lncRNAs and 56 mRNAs, indicating that pairing did not occur exclusively in a one-to-one manner. No Lnc-CompleteIn-mRNA exon pairs were found (Fig. 4; Additional file 7).

\section{IncRNA-mRNA pairs involved in floral transition}

To better understand the roles of lncRNAs, we analysed the lncRNA-mRNA interactions and predicted the lncRNA protein-coding targets (Additional file 7). Seven of the mRNAs were related to flowering: Cbu.gene.18564, Cbu.gene.2167, Cbu.gene.190, Cbu.gene.24144, Cbu.gene.7250, Cbu.gene.20428, and Cbu.gene.1092 (Table 3). To further study the correlations of the lncRNA-mRNA pairs, we analysed the correlation coefficients of lncRNAs and mRNAs between the EF and NF samples during three developmental periods. In-depth research was performed on three lncRNAs-mRNAs pairs: LXLCO_019079/Cbu.gene.1092, LXLOC_017817/Cbu.gene.24144 and LXLOC_030659/ Cbu.gene.7250. The expression trends of the mRNAs were highest in EF-Tbs. These results were consistent with the expression patterns of the homologous genes in Arabidopsis [31, 33]. Based on the overall trend, the three pairs exhibited negative correlations with $r<-0.6$. While the observed expression correlations between lncRNAs and their co-expressed genes are highly intriguing, whether they reflect true regulatory relationships requires further testing (Fig. 5).

\section{GO analysis of DEGs and target genes of DELs}

In total, 680 DEGs and 817 DELs were identified in NF-Vbs versus EF-Vbs, 1089 DEGs and 1087 DELs were identified in NF-Tbs versus EF-Tbs, and 306 DEGs and 514 DELs were identified in NF-Rbs versus EF-Rbs (Fig. 6, Additional file 8). Fig. 6b shows the numbers of up- or down-regulated mRNAs and lncRNAs.

Eighteen DEGs related to flower development were identified in all three groups (Table 4). However, genes regulating the vegetative-reproductive transition were identified only in NF-Vbs versus EF-Vbs. These genes included one SPL gene (Cbu.gene.13552) and two novel genes (Cbu.gene.5215 and Cbu.gene.12342). Cbu.gene.13552, which

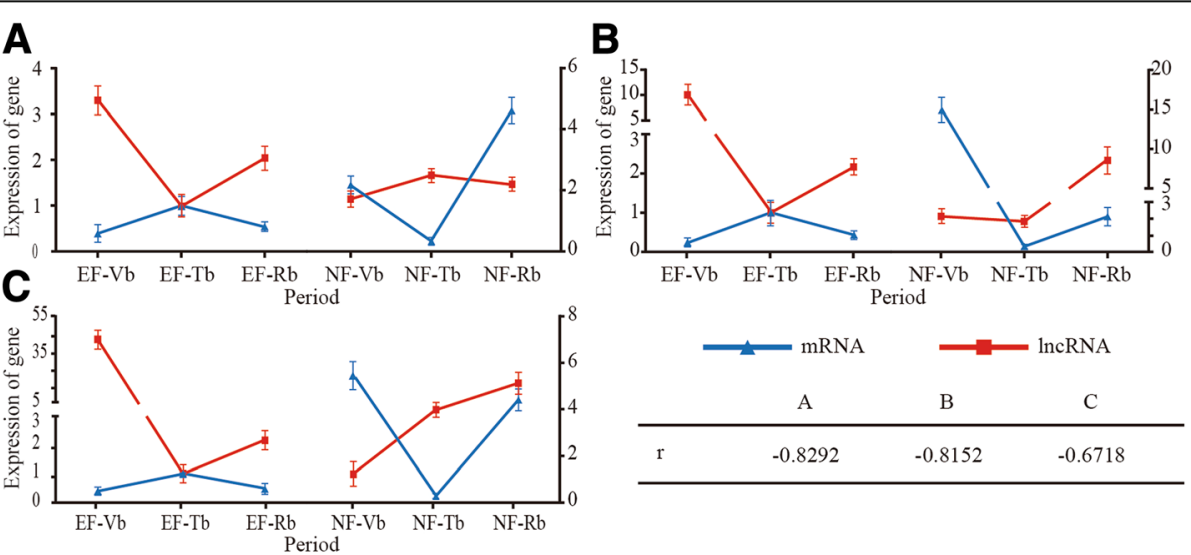

Fig. 5 The correlation coefficients of expression of IncRNAs and mRNAs between the EF and NF varieties during three developmental periods. a The expression of LXLOC.019079-Cbu.gene.1092 between the EF and NF varieites during the three developmental periods. b The expression of LXLOC.017817Cbu.gene.24144 between the EF and NF varieties during the three developmental periods. c The expression of LXLOC.030659-Cbu.gene.7250 between the $\mathrm{EF}$ and NF varieties during the three developmental periods. The red line with a square represents the expression of IncRNA, and the blue line with a triangle represents the expression of mRNA. $r$ is the correlation coefficient of expression of the three "co-expression" pairs 

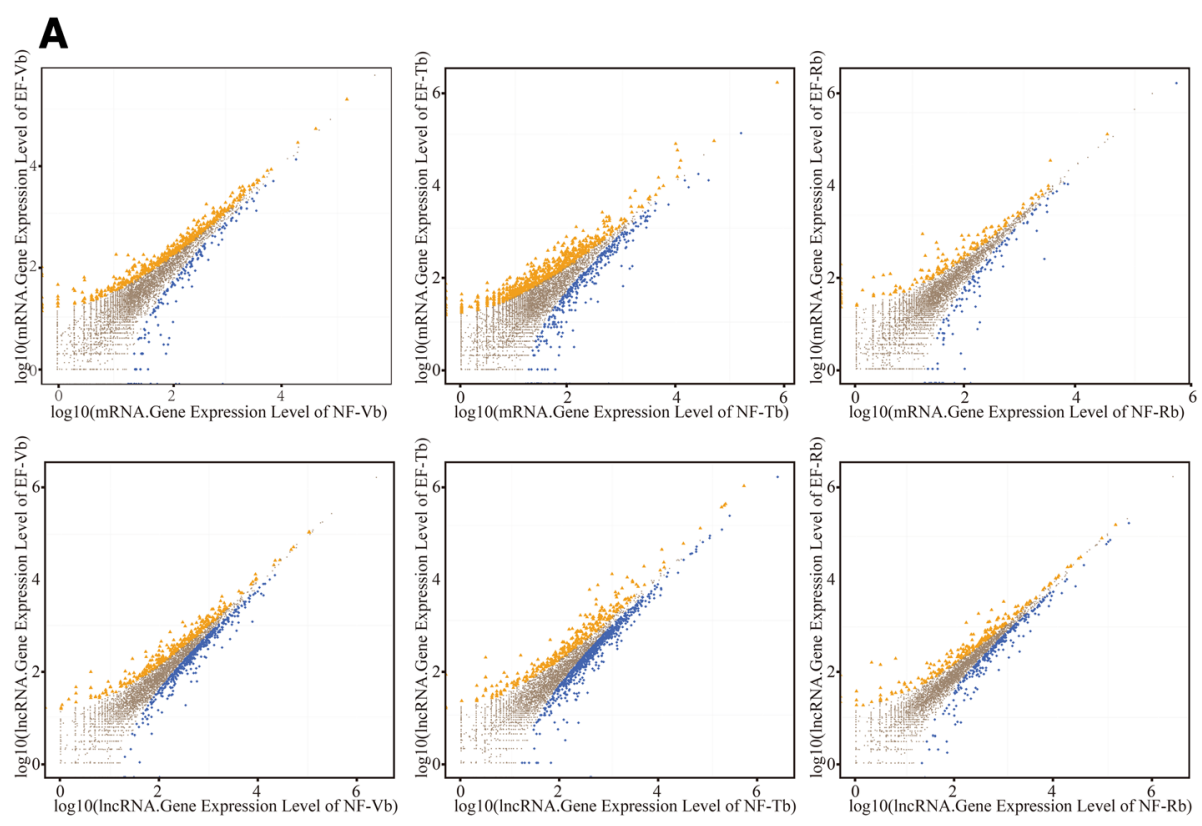

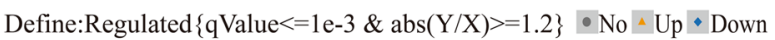

$\underline{B}$

\begin{tabular}{lcccc}
\hline & \multicolumn{2}{c}{ DEGs } & \multicolumn{2}{c}{ DELs } \\
\cline { 2 - 5 } & Up & Down & Up & Down \\
\hline NF-Vb vs EF-Vb & 525 & 155 & 330 & 487 \\
NF-Tb vs EF-Tb & 723 & 357 & 319 & 768 \\
NF-Rb vs EF-Rb & 172 & 155 & 246 & 268 \\
\hline
\end{tabular}

Fig. 6 DEGs and DELS in NF and EF C. bungei. a Scatter plot of the comparative results of the log-transformed gene expression levels (Log10 FPKM) and DEG or DEL distributions between the NF and EF samples. The orange rectangles represent up-regulated genes, and the blue diamonds represent down-regulated genes. $\mathbf{b}$ Summary of the numbers of up- or down-regulated mRNAs and IncRNAs in NF-Vb vs. EF-Vb, NF-Tb vs. EF-Tb, and NF-Rb vs. EF-Rb

exhibited greater expression in EF-Vbs than in NF-Vbs, was predicted to be a target of miR529 (psRNATarget, Additional file 9), which is consistent with previous reports of SPL [42]. These results suggested that, although the morphological shift of the floral transition could only be observed in EF-Tbs, the floral transition had started in EF-Vbs at a molecular level. Overall, the $\mathrm{Vb}$ phase is a critical stage for floral transition gene regulation.

\section{DEGs involved in floral transition}

Floral transition has been studied extensively in Arabidopsis thaliana and more than 100 floral transition-related genes have been reported (Fig. 7, Additional file 1). Many homologous genes related to floral transition were found by the BLAST search against the transcriptome data. In addition to the aforementioned genes, several additional genes related to flowering were differentially expression in EF and
NF buds (Fig. 8, Additional file 10). Five SPL homologous genes were found in this study, and these genes exhibited greater expression in EF-Vbs than in NF-Vbs. SPLs include very large gene families that mainly promote flowering time via age pathways $[43,44]$. SPLs promote floral transition by activating the expression of several other genes, such as FT genes.

Cbu.gene.26021 is homologous with genes in the FT family. Cbu.gene.26021 exhibited substantially greater expression in EF versus NF buds at all stages. FT and TSF are FT gene family members that work redundantly to promote floral transition in Arabidopsis [45]. These results imply that $F T$ family-associated flowering transition regulation may exist in $C$. bungei.

The DELLA homologous genes, except for Cbu.gene.10036, exhibited greater expression in EF buds than in NF buds. In Arabidopsis, DELLA is a negative flowering 
Table 4 Parital Gene ontology associated with flower development

\begin{tabular}{|c|c|c|}
\hline Gene ID & Term & GO ID \\
\hline \multirow[t]{3}{*}{ Cbu.gene.11993 } & floral whorl development & GO:0048438 \\
\hline & floral organ development & GO:0048437 \\
\hline & flower development & GO:0009908 \\
\hline Cbu.gene.13552 & regulation of flower development & GO:0009909 \\
\hline \multirow[t]{3}{*}{ Cbu.gene.16262 } & pollen development & GO:0009555 \\
\hline & pollen exine formation & GO:0010584 \\
\hline & pollen wall assembly & GO:0010208 \\
\hline Cbu.gene.16343 & flower development & GO:0009908 \\
\hline Cbu.gene.17110 & pollen development & GO:0009555 \\
\hline \multirow[t]{3}{*}{ Cbu.gene.17779 } & recognition of pollen & GO:0048544 \\
\hline & pollen-pistil interaction & GO:0009875 \\
\hline & flower development & GO:0009908 \\
\hline Cbu.gene.18350 & floral organ development & GO:0048437 \\
\hline \multirow[t]{3}{*}{ Cbu.gene.20397 } & pollen development & GO:0009555 \\
\hline & pollen exine formation & GO:0010584 \\
\hline & pollen wall assembly & GO:0010208 \\
\hline Cbu.gene.20428 & flower development & GO:0009908 \\
\hline \multirow[t]{3}{*}{ Cbu.gene.21602 } & flower development & GO:0009908 \\
\hline & floral whorl development & GO:0048438 \\
\hline & floral organ development & GO:0048437 \\
\hline \multirow[t]{2}{*}{ Cbu.gene.24436 } & recognition of pollen & GO:0048544 \\
\hline & pollen-pistil interaction & GO:0009875 \\
\hline \multirow[t]{4}{*}{ Cbu.gene.24775 } & floral whorl development & GO:0048438 \\
\hline & floral organ development & GO:0048437 \\
\hline & stamen development & GO:0048443 \\
\hline & androecium development & GO:0048466 \\
\hline Cbu.gene.26021 & flower development & GO:0009908 \\
\hline Cbu.gene. 5215 & regulation of flower development & GO:0009909 \\
\hline \multirow[t]{3}{*}{ Cbu.gene.6097 } & pollen exine formation & GO:0010584 \\
\hline & pollen wall assembly & GO:0010208 \\
\hline & pollen development & GO:0009555 \\
\hline Cbu.gene.6356 & shoot system development & GO:0048367 \\
\hline Cbu.gene.8663 & flower development & GO:0009908 \\
\hline \multirow[t]{4}{*}{ Cbu.gene.26687 } & floral whorl development & GO:0048438 \\
\hline & floral organ development & GO:0048437 \\
\hline & stamen development & GO:0048443 \\
\hline & androecium development & GO:0048466 \\
\hline
\end{tabular}

regulation gene that has been found to show lower expression levels in EF buds than in NF buds [46]. Therefore, Cbu.gene.10036 may be the DELLA gene in C. bungei, or $D E L L A$ homologs may have a novel role in floral transition in C. bungei (Fig. 8, Additional file 10).
Relationships between DELs and miRNA families

The DELs were distributed in 437 families, including 34 known miRNA families (Fig. 9, Additional file 11). These 34 families included 466 related DELs, which may act as decoys of the corresponding miRNAs. The DELs LXLOC_012080, LXLOC_017995, LXLOC_ 031572, LXLOC_019956, LXLOC_009916, LXLOC_ 007700, and LXLOC_008874 were related to more than one of the miRNA families miR2118, miR529, miR535, miR408, and miR390 (Fig. 9a, inner layers). In particular, LXLOC_019956 was related to all five miRNA families. These miRNA families are associated with resistance, floral transition, and sucrose metabolism. Most of the lncRNAs were related to unique miRNA families (Fig. 9a, outer layer, B). Together, the DELs and miRNA families formed a complex network to regulate plant development and growth. The relationships between the DELs and miRNA families imply the involvement of plant resistance and sucrose metabolism in floral transition.

\section{Expression trends of the selected DEGs and DELs in EF and NF buds}

We performed RNA-seq and qRT-PCR analysis on five lncRNAs and six mRNAs in NF and EF buds from three growth periods (Fig. 10). The five DELs were related to the five flowering-related miRNA families: miR408, miR398, miR529, miR535, and miR159. The six DEGs were SPL, bHLH, LFY, CONTANS, and STP (sugar transport protein). The expression trends can be summarized into three categories. I) The expression levels of some lncRNAs or mRNAs, such as LXLOC_010612, LXLOC_ 022980, Cbu.gene.21740, and Cbu.gene.21762, showed different trends in the NF and EF buds. In the NF buds, LXLOC_010612, which is related to miR529, was highly expressed in Vbs, but LXLOC_010612 expression was decreased in Tbs, and higher levels of LXLOC_010612 were restored in Rbs. This gene showed the opposite expression pattern in EF buds. In EF buds, LXLOC_ 010612 showed the highest expression level in Tbs and lower expression levels in Vbs and Rbs. Cbu.gene.21762 was a presumed SPL family gene. No obvious expression changes were noted in NF/EF-Vbs and NF/EF-Tbs; however, in NF-Rbs, the expression level of Cbu.gene.21762 increased sharply. This increase was minimal in EF buds. Cbu.gene.21740 is a $b H L H$ family gene reportedly involved in the jasmonate signalling pathway of plants [47]. From the $\mathrm{Vb}$ to $\mathrm{Tb}$ phases, the expression of Cbu.gene. 21740 decreased in EF buds but increased slightly in NF buds. The different gene expression trends between the EF and NF buds suggest that these genes have a role in the early flowering phenomenon of the EF variety. II) The expression levels of some lncRNAs or mRNAs, such as LXLOC_009916, LXLOC_00700, 


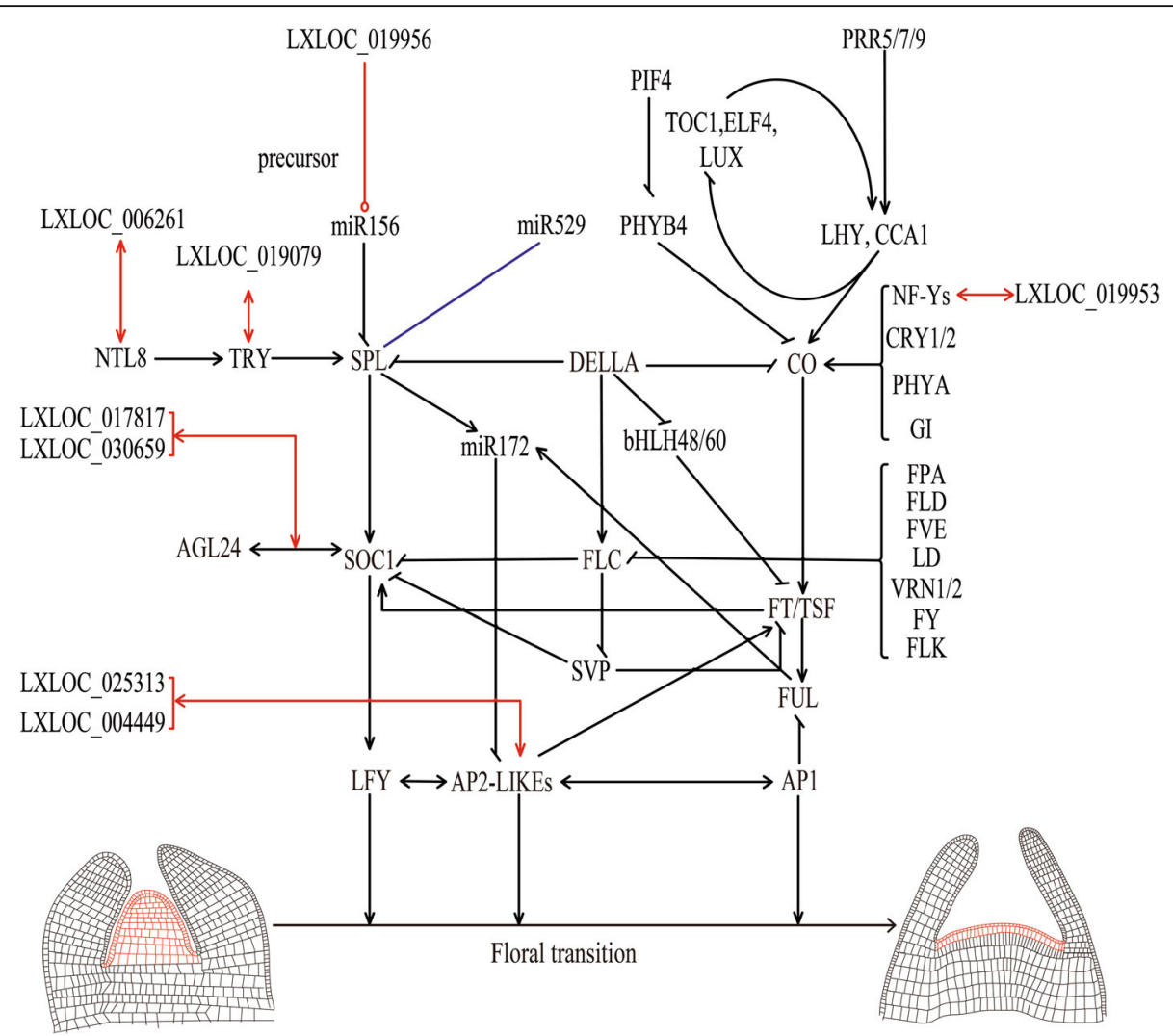

Fig. 7 Floral transition network in Arabidopsis. The red text represents the main genes in the floral transition network of Arabidopsis. The black lines represent the relationships in Arabidopsis. The red lines represent the relationships implied by this study in C. bungei. The singe-headed arrows indicate that gene expression is promoted. The double-headed arrows indicate the IncRNA-mRNA pairs in C. bungei. The curly brackets with arrows indicate that the expression of the genes in the brackets is promoted. The short lines indicate negative gene expression. The red lines with rings indicate gene precursors. The bottom figure shows the floral transition. In this bottom figure, the red areas represent the shoot apical meristem, the left image shows the vegetative growth terminus, and the right image shows the reproductive growth terminus

Cbu.gene.4037, Cbu.gene.14754, and Cbu.gene.21122, showed the same trends in NF and EF buds. III) The expression levels of some lncRNAs or mRNAs showed no obvious trends. Overall, the qRT-PCR and RNA-seq results were in good agreement. The expression levels of the lncRNAs and mRNAs showed period-specific patterns. The mRNAs or lncRNAs that were differentially expressed in the NF and EF buds may contribute to early flowering in the EF variety.

\section{Discussion}

C. bungei is an ancient ornamental woody plant in China. The flowering time of this species largely contributes to its commercial value. Floral transition represents a major developmental phase change that transforms the identity of the shoot apical meristem from vegetative to inflorescence. However, partly due to the difficulty associated with selecting the EF buds of $C$. bungei, few studies have investigated the flowering transition of this species. With the development of RNA-seq technology, genome-wide mapping has proven to be a powerful tool for studying the flowering transition in C. bungei. Here, we present the first comprehensive analysis of the lncRNAs in C. bungei to study floral transition regulation in woody plants. The transcriptomic study was performed with NF and EF varieties to systematically identify the lncRNAs and mRNAs associated with floral transition. In total, 12,532 lncRNAs and 26,936 mRNAs were detected, including 680 DELs and 817 DEGs. Seven lncRNAs were predicted to be miRNA precursors. In addition, 119 IncRNA-mRNA interaction pairs were identified based on localization, function prediction, and binding energy analyses. The results suggest that the molecular regulation of floral transition may occur before the associated morphological changes. Our results offer a deeper understanding of the floral transition mechanism in C. bungei, and the selected lncRNAs represent potential targets for future studies.

In this study, most mRNAs, such as $S P L, A P 2, L F Y$, $\mathrm{CO}$, showed expression patterns similar to those in Arabidopsis [30, 34, 36, 37, 48, 49]. Cbu.gene.13552, an SPL homologous gene, generally showed high expression 


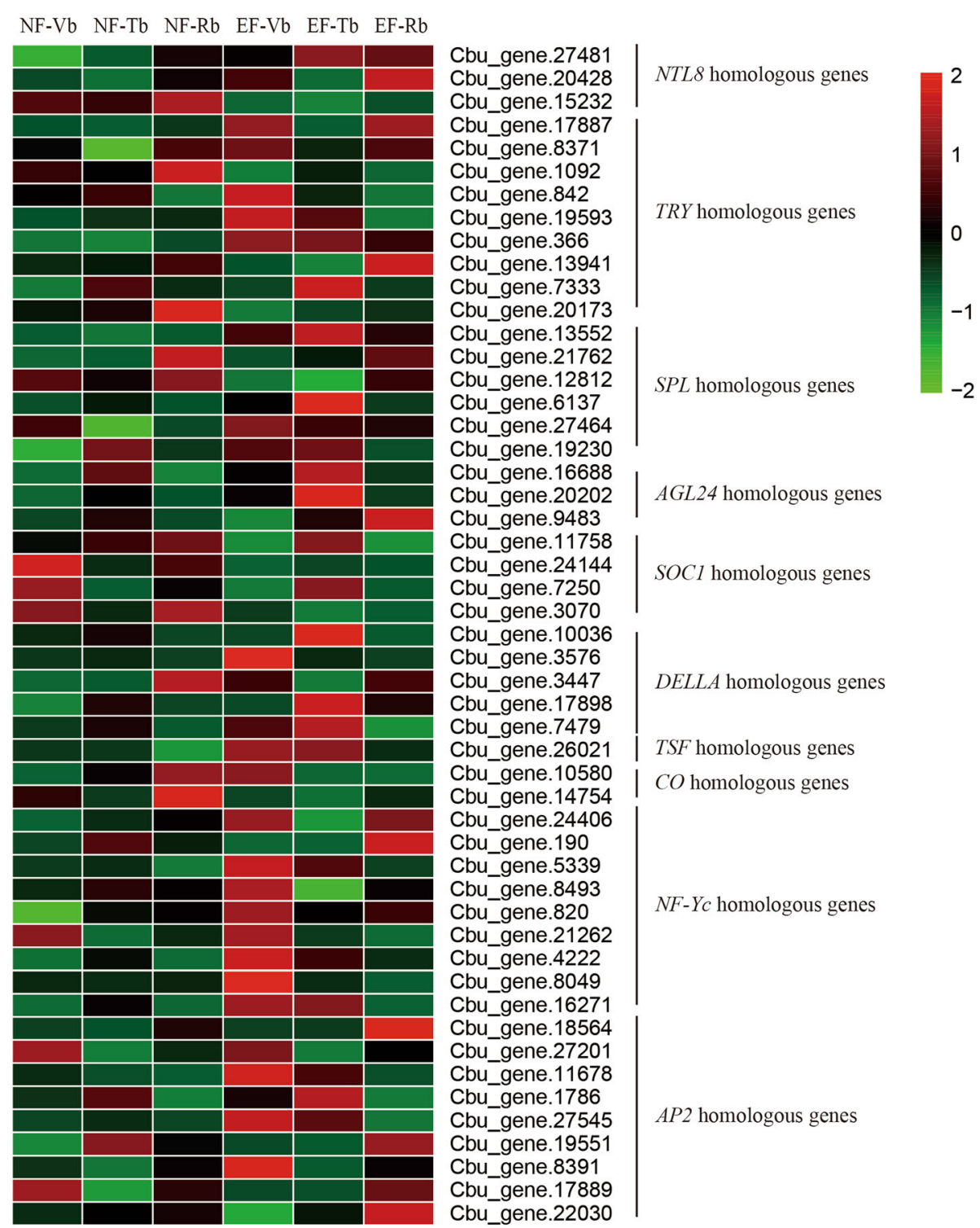

Fig. 8 Expression analysis of the floral transition homologous genes in C. bungei. Vertical bars indicate homologous genes from Arabidopsis. The text alternates between black and red for clarity

levels in Vbs. In addition, after a short period of decline, the expression levels of this gene increased during the flower formation stage. Cbu.gene.13552 showed expression trends in C. bungei similar to those of SPLs in Arabidopsis (Fig. 8) [35, 43, 49, 50]. However, the expression trends of some genes in C. bungei differed from those of corresponding genes in Arabidopsis. For example, except for Cbu.gene.10036, DELLA homologous genes in C. bungei exhibited greater expression in EF buds than in NF buds. In Arabidopsis, DELLA is a negative flowering regulation gene that has been shown to exhibit lower expression levels in EF versus NF buds [46, 51]. FT and TSF are important genes related to floral transition in
Arabidopsis that regulate floral transition in response to day length (Fig. 8, Additional file 10) [52, 53]. Cbu.gene.18536 is a possible homolog of $F T$, but no differences in the expression levels of this gene were detected between EF and NF buds. Meanwhile, Cbu.gene.26021, a possible homolog of TSF, showed a markedly higher expression level in EF buds than in NF buds.

Two main reasons account for these differences in gene expression between Arabidopsis and C. bungei. First, the sequences and functions of genes in herbs and woody plants may differ. Second, because genes from the same family may have different functions, genes may be annotated inaccurately by imperfect genome annotation information. 


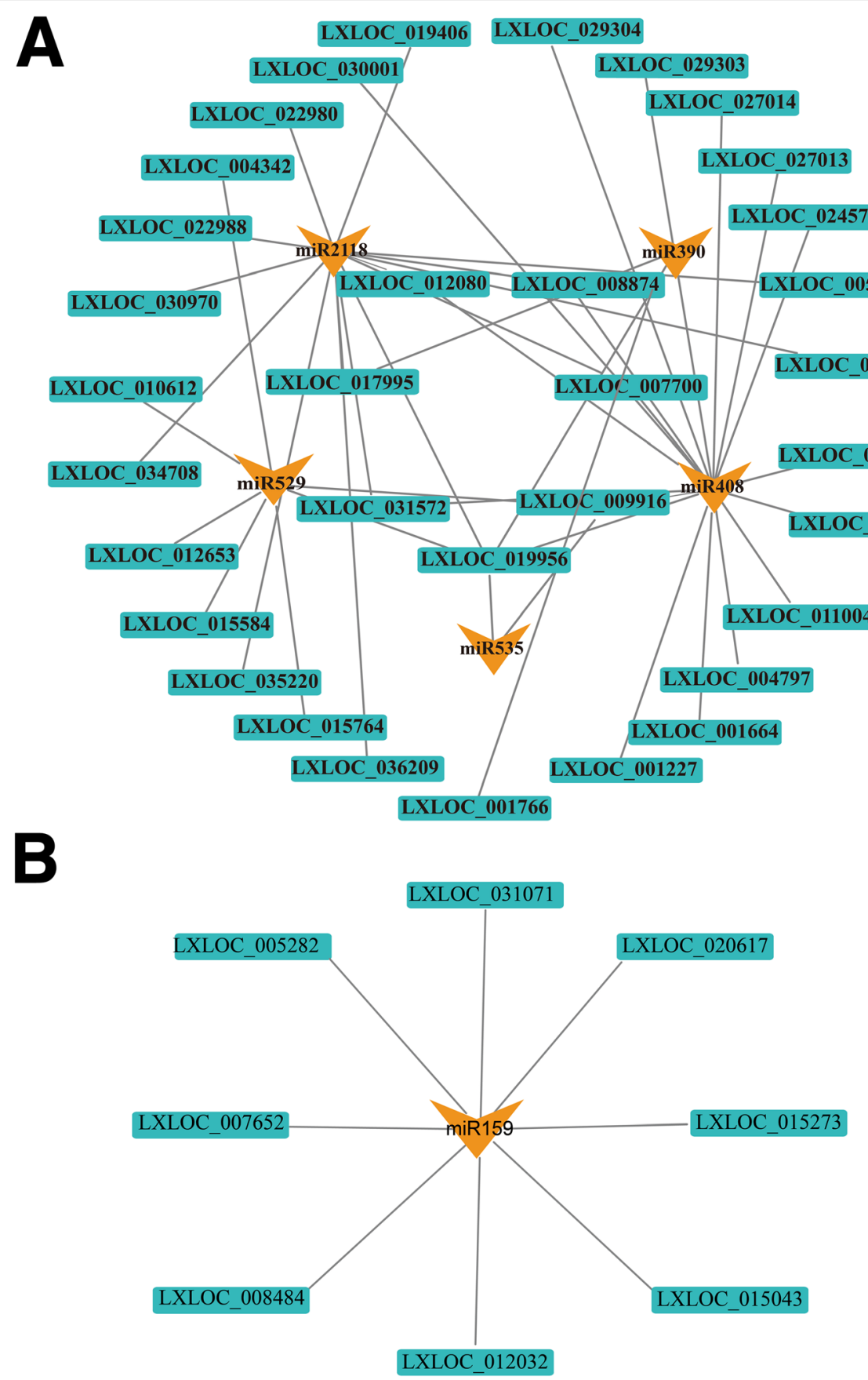

Fig. 9 Relationship network of IncRNAs and miRNAs. INFERNAL (http://eddylab.org/infernal/) was used to perform a BLAST search with the IncRNAs of the Rfam database (http://rfam.xfam.org/). In total, 466 IncRNAs may act as decoys of the corresponding miRNAs. The orange triangles indicate the miRNA families. The blue rectangles represent the related IncRNAs. The relationships between the IncRNAs and miRNAs are diverse. a Several IncRNAs have relationships with more than one miRNA family. The rings, from outside inward, represent IncRNAs that do not overlap, miRNA families, and IncRNAs that overlap. b Nine IncRNAs show a relationship with miR159

In this study, seven lncRNAs were identified as precursors of miRNAs, including three known lncRNAs and four novel lncRNAs (Table 2). LXLOC_019956 represents a potentially intriguing case. The IncRNA LXLOC_019956 was a precursor of miR156 (Additional file 6). According to existing reports, miR156 targets the SPL family of genes during the flowering transition $[35,43,49,54,55]$. Therefore, LXLOC_019956 may indirectly participate in the floral transition process (Fig. 3).

Moreover, LXLOC_019956 is related to miR529/535/ $408 / 390 / 2118$, which are crucial for plant growth and resistance (Fig. 9). miR529/535 have a similar function as 

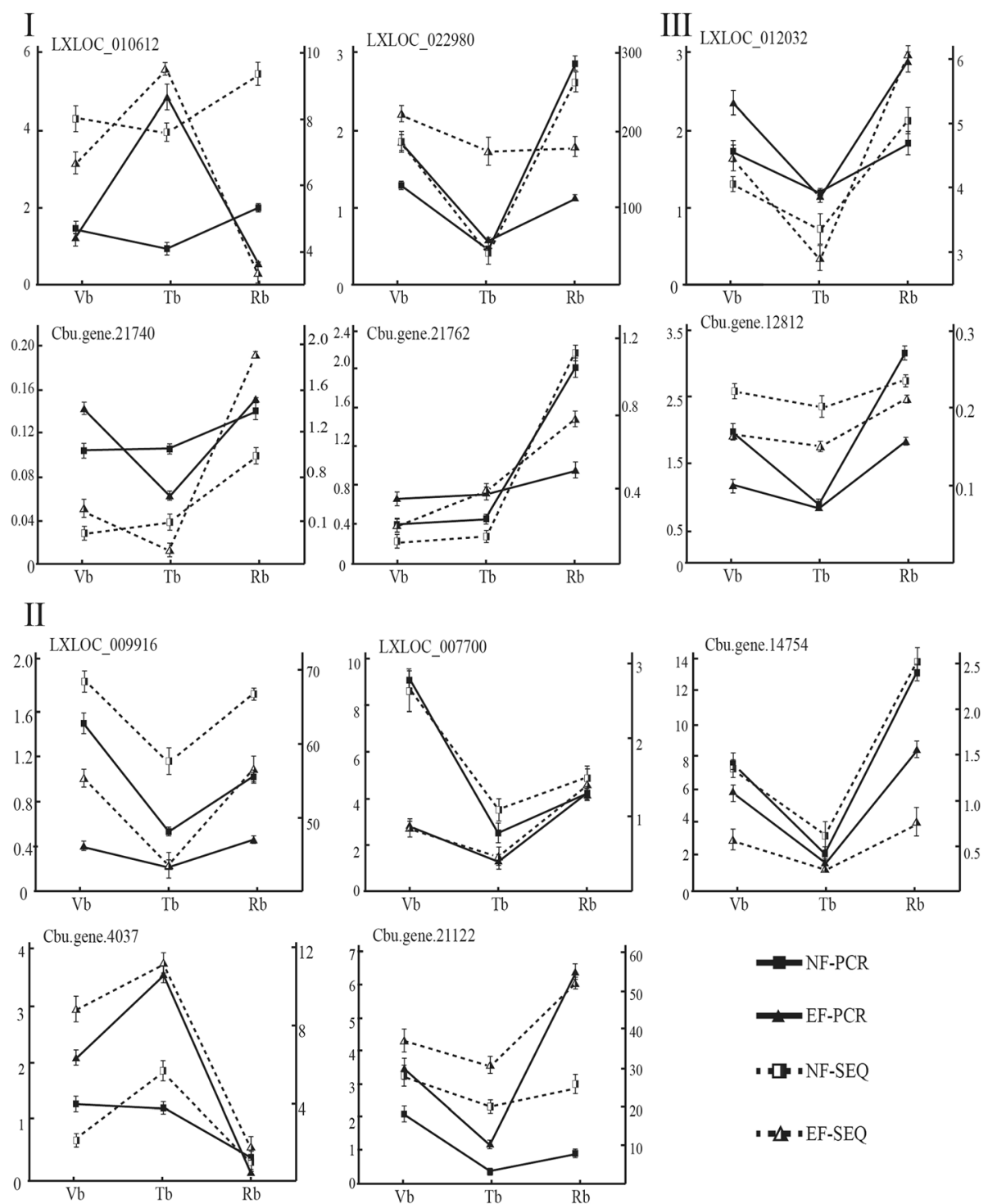

Fig. 10 IncRNA and mRNA results from RNA-seq verified by qRT-PCR. i IncRNA or mRNA expression levels showing different trends in NF and EF buds; ii IncRNA or mRNA expression levels showing the same trend in NF and EF buds; iii IncRNA or mRNA expression showing no obvious different trend. The $\mathrm{Y}$-axis represents the relative expression of genes, and the $\mathrm{X}$-axis represents the development periods. $\rightarrow-$ represents the PCR results in NF buds, $\longrightarrow$ represents the PCR results in EF buds, - $\mathbf{\square}$ - represents the RNA-seq results in NF buds, and - $\mathbf{-} \mathbf{-}-$ represents the RNA-seq results in EF buds

miR156 [42, 54, 55]. Cbu.gene.13552, a possible SPL gene that was significantly up-regulated in EF-Vbs, was predicted to be a target of miR529 (Additional file 9). Regulation of specific SPLs by miR156 and miR529 is important in flower architecture development in monocots, particularly in grasses. However, in this study, miR529s were present in the eudicot C. bungei.

In this study, we identified 119 lncRNA-mRNA pairs (Additional file 7). Seven mRNAs from these pairs are encoded by homologs of genes related to floral transition, including SOC1s, AP2s, NTL8, TRY, and NF-YC (Fig. 7). According to reports, IncRNAs with enhancer-like functions have been discovered, and these lncRNAs also serve as NATs to inhibit the expression of the corresponding mRNAs $[21,56]$. Based on the concept that lncRNAs and their regulatory targets may exhibit highly positively or negatively correlated expression patterns, we analysed the expression correlations of lncRNAs and their targets. In our study, we showed three examples of IncRNAs and their negatively correlated mRNAs, including LXLOC_019079 and Cbu.gene.1092 (TRY homologous gene), LXLOC_ 017817 and Cbu.gene.24144 (SOC1 homologous gene) and LXLOC_030659 and Cbu.gene.7250 (SOC1 homologous gene) (Fig. 5). The three homologous genes promoted the 
floral transition in Arabidopsis [31]. The identification of three lncRNAs with opposite expression patterns relative to those of their targets warrants further investigation into a possible direct regulatory relationship between lncRNAs and their targets. These results suggest that floral transition-repressive lncRNAs may serve as the hubs of a gene regulatory network, the suppression of which may lead to positive vegetative growth and material development. Overall, lncRNAs have complex functions in organisms. More tests should be carried out to verify the functions of lncRNAs and their mechanisms of action.

We examined the expression levels of five lncRNAs and six mRNAs by qRT-PCR and RNA-seq (Fig. 10). The expression trends of LXLOC_010612, a bHLH gene (Cbu.gene.21740), and an SPL gene (Cbu.gene.21762) differed significantly between NF and EF buds. LXLOC_ 010612 was related to miR529, which is important in floral transition. SPLs participate in many biological and metabolic processes, such as resistance and floral transition. The expression results suggested that LXLOC_010612, the $b H L H$ gene, and the SPL gene may be involved in early flowing in the EF variety. However, these genes should be further studied to determine whether they are related to floral transition or flower development.

\section{Conclusions}

In this manuscript, we recorded the external and internal morphologies of EF and NF buds in different growth phases. In this study, 12,532 lncRNAs and 26,936 messenger RNAs (mRNAs) were detected. In addition, 680 DEGs and 817 differentially expressed lncRNAs were detected during the initiation of floral transition. Moreover, the lncRNA LXLOC_019956 was a precursor of miR156, which targets the SPL family of genes to enable flowering transition. In addition, seven lncRNA-mRNA interaction pairs were predicted to be involved in floral transition. The present study provides new insights into the role of lncRNAs in the molecular mechanisms underlying the flowering transition. These results can be used to explore the functions of lncRNAs and support further genetic studies of $C$. bungei.

\section{Additional files}

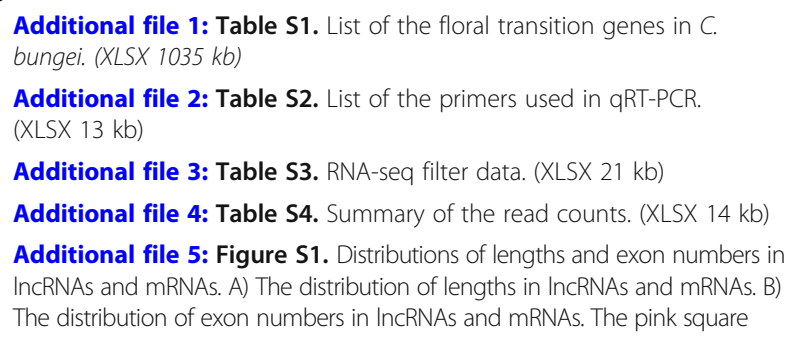

represents the distribution in IncRNAs and the blue and red squares represent the distribution in mRNAs. (JPG $6734 \mathrm{~kb}$ )

Additional file 6: Table S5. miRNA precursor prediction. (XLSX $10 \mathrm{~kb}$ ) Additional file 7: Table $\mathrm{S6}$. Prediction of the localization relationships of IncRNAs and mRNAs. (XLSX $19 \mathrm{~kb}$ )

Additional file 8: Table S7. Summary of the DEGs and DELs in different groups. (XLSX $11 \mathrm{~kb}$ )

Additional file 9: Figure S2. Alignment of Cbu.gene.13552 with miR529. (JPG 1754 kb)

Additional file 10: Figure S3. Expression analysis of the homologous genes involved in floral transition in C. bungei. The vertical bar indicates that the gene is the homologous gene from Arabidopsis. Black red alternation is present to prevent disorder. (JPG 5059 kb)

Additional file 11: Figure S4. Interaction networks of IncRNAs and miRNAs. The orange triangle indicates the miRNA families The blue rectangle indicates the related IncRNAs. The relationships among the IncRNAs and miRNAs are diverse. A) The IncRNAs and miRNA families have intersecting relationships. B) The relationships between with the IncRNAs and miRNA families are 1 to a model. C) The relationships between certain IncRNAs and miRNAs intersect. (JPG $4588 \mathrm{~kb}$ )

\section{Abbreviations}

AGL24: Agamous-like 24; AP1: Apetala 1; AP2: Apetala 2; bHLH: Basic helixloop-helix; CCA1: Circadian Clock Associated1; CO: Constans; CRY: Cryptochrome; DEG: Differentially expressed gene; DEL: Differentially expressed LncRNA; EF: Early flowering mutant; ELF: Early flowering; FD: Flowering locus D; fl: Flower primordial; FLC: Flowering Locus C; FPKM: Fragments mapped; FRI: Frigida; FT: Flowering locus T; Ga: Generative apex; Gl: Gigantea; Ip: Leaf primordium; LFY: Leafy; LHY: Latelongated Hypocoty L; LncRNA: Long non-coding RNA;

LUX: Luxarrhythmo; miRNA: MicroRNA; MYB: Myeloblastosis viral oncogene homolog; NAT: Natural antisense transcript; NF: Normal flowering; NF-Ys: Nuclear factor-YS; NTL: NAC with transmembrane motif 1 like; PHYB: PhytochromeB; PIF: Phytochrome interacting factor; Rb: Reproductive bud; SOC1: Suppressor of overexpression of CO1; SPL: Squamosa-promoter binding protein-like; sRNA: Small RNA; SVP: Short vegetative phase; Tb: Transition bud; TRY: Triptychon; TSF: Twin sister of FT; Vb: Vegetative bud; VRN: Vernalization

\section{Acknowledgements}

We would like to thank the laboratory for participating in the study. We acknowledge the anonymous referees for their constructive comments on the paper, which have greatly improved its presentation.

\section{Funding}

This work was supported by Forestry Industry Research Special Funds for Public Welfare Projects [201504101]. The funders had no role in study design, data collection and analysis, decision to publish, or preparation of the manuscript.

\section{Availability of data and materials}

The complete clean reads have been uploaded to the SRA site (http:// www.ncbi.nlm.nih.gov/sra/). Data access: SRP120718 in NCBI.

\section{Authors' contributions}

JHW, GZQ and ZW designed the experiments. ZW analyzed the RNA-seq data and wrote the manuscript. ZW and TQZ detected the expression of genes using qRT-PCR. ZW, WJM and NW collected the samples used in the experiment. All the authors have read the paper and agreed to list their names as coauthors. All authors read and approved the final manuscript.

Ethics approval and consent to participate

All the plant materials used in this study were provided by Research Institute of Forestry, Chinese Academy of Forestry. The field experiments were conducted under local legislation and permissions.

Consent for publication Not applicable. 


\section{Competing interests}

The authors declare that they have no competing interests.

\section{Publisher's Note}

Springer Nature remains neutral with regard to jurisdictional claims in published maps and institutional affiliations.

\section{Author details}

'State Key Laboratory of Tree Genetics and Breeding, Key Laboratory of Tree Breeding and Cultivation of State Forestry Administration, Research Institute of Forestry, Chinese Academy of Forestry, Haidian District, Dongxiaofu 1\#, Beijing 100091, People's Republic of China. ${ }^{2}$ State Key Laboratory of Tree Genetics and Breeding (Northeast Forestry University), 26 Hexing Road, Harbin 150040, People's Republic of China.

Received: 19 December 2017 Accepted: 10 September 2018 Published online: 20 September 2018

\section{References}

1. Guo X, Gao L, Wang Y, Chiu DKY, Wang T, Deng Y. Advances in long noncoding RNAs: identification, structure prediction and function annotation. Brief Funct Genomics. 2016;15:38-46.

2. Xing Y-H, Bai Z, Liu C-X, Hu S-B, Ruan M, Chen L-L. Research progress of long noncoding RNA in China. IUBMB Life. 2016;68:887-93 Available from: http://doi.wiley.com/10.1002/iub.1564.

3. Penny GD, Kay GF, Sheardown SA, Rastan S, Brockdorff N. Requirement for Xist in X chromosome inactivation. Nature. 1996;379:131.

4. Kung JTY, Colognori D, Lee JT. Long noncoding RNAs: past, present, and future. Genetics. 2013;193:651-69 Available from: http://www.genetics.org/ cgi/doi/10.1534/genetics.112.146704.

5. Bai $Y$, Dai $X$, Harrison AP, Chen M. RNA regulatory networks in animals and plants: a long noncoding RNA perspective. Brief Funct Genomics. 2015;14: 91-101.

6. Chen M, Wang C, Bao H, Chen H, Wang Y. Genome-wide identification and characterization of novel IncRNAs in Populus under nitrogen deficiency. Mol Gen Genomics. 2016:291:1663-80.

7. Wang H, Chung PJ, Liu J, Jang IC, Kean MJ, Xu J, et al. Genome-wide identification of long noncoding natural antisense transcripts and their responses to light in Arabidopsis. Genome Res. 2014;24:3.

8. Chen J, Quan M, Zhang D. Genome-wide identification of novel long noncoding RNAs in Populus tomentosa tension wood, opposite wood and normal wood xylem by RNA-seq. Planta. 2015;241:125-43 Available from: http://link.springer.com/10.1007/s00425-014-2168-1.

9. Zhu B, Yang Y, Li R, Fu D, Wen L, Luo Y, et al. RNA sequencing and functional analysis implicate the regulatory role of long non-coding RNAs in tomato fruit ripening. J Exp Bot. 2015;66:4483-95.

10. Ausin I, Greenberg MVC, Simanshu DK, Hale CJ, Vashisht AA, Simon SA, et al. INVOLVED IN DE NOVO 2-containing complex involved in RNA-directed DNA methylation in Arabidopsis. Proc Natl Acad Sci. 2012;109:8374-81 Available from: http://www.pnas.org/cgi/doi/10.1073/pnas.1206638109.

11. Chen $X, \mathrm{Hu}$ J, Zhang $H$, Ding Y. DNA methylation changes in photoperiodthermo-sensitive male sterile rice PA64S under two different conditions. Gene. 2014;537:143-8.

12. Wang Z-W, Wu Z, Raitskin O, Sun Q, Dean C. Antisense-mediated FLC transcriptional repression requires the P-TEFb transcription elongation factor. Proc Natl Acad Sci. 2014;111:7468-73 Available from: http://www. pnas.org/cgi/doi/10.1073/pnas.1406635111.

13. Marquardt S, Raitskin O, Wu Z, Liu F, Sun Q, Dean C. Functional consequences of splicing of the antisense transcript COOLAIR on FLC transcription. Mol Cell. 2014;54:156-65 Available from: https://doi.org/10. 1016/j.molcel.2014.03.026.

14. Ding J, Lu Q, Ouyang Y, Mao H, Zhang P, Yao J, et al. A long noncoding RNA regulates photoperiod-sensitive male sterility, an essential componen of hybrid rice. Proc Natl Acad Sci. 2012;109:2654-9 Available from: http:// www.pnas.org/cgi/doi/10.1073/pnas.1121374109.

15. Heo JB, Sung S. Vernalization-mediated epigenetic silencing by a long intronic noncoding RNA. Science. 2011:331:76

16. Swiezewski S, Liu F, Magusin A, Dean C. Cold-induced silencing by long antisense transcripts of an Arabidopsis Polycomb target. Nature. 2009:462: 799-802 Available from: https://doi.org/10.1038/nature08618.
17. Bryan JHD. Differential staining with a mixture of safranin and fast green FCF. Stain Technol. 1955:30:153-7.

18. Jing D, Xia Y, Chen F, Wang Z, Zhang S, Wang J. Ectopic expression of a Catalpa bungei (Bignoniaceae) PISTILLATA homologue rescues the petal and stamen identities in Arabidopsis pi-1 mutant. Plant Sci. 2015;231:40-51 Available from: https://doi.org/10.1016/j.plantsci.2014.11.004.

19. Secco D, Jabnoune $M$, Walker $H$, Shou H, Wu P, Poirier $Y$, et al. Spatiotemporal transcript profiling of rice roots and shoots in response to phosphate starvation and recovery. Plant Cell. 2013;25:4285-304 Available from: http://www.plantcell.org/cgi/doi/10.1105/tpc.113.117325.

20. Li CY, Deng GM, Yang J, Viljoen A, Jin Y, Kuang RB, et al. Transcriptome profiling of resistant and susceptible Cavendish banana roots following inoculation with Fusarium oxysporum f. sp. cubense tropical race 4. BMC Genomics. 2012;13:1 Available from: BMC Genomics.

21. Chen M, Xu R, Ji H, Greening DW, Rai A, Izumikawa K, et al. Transcriptome and long noncoding RNA sequencing of three extracellular vesicle subtypes released from the human colon cancer LIM1863 cell line. Sci Rep. 2016:6:114 Available from: https://doi.org/10.1038/srep38397.

22. Kong $L$, Zhang $Y$, Ye ZQ, Liu XQ, Zhao SQ, Wei $L$, et al. CPC: assess the protein-coding potential of transcripts using sequence features and support vector machine. Nucleic Acids Res. 2007:35:W345-349.

23. Satovic Z, Avila CM, Cruz-lzquierdo S, Díaz-Ruíz R, García-Ruíz GM, Palomino C, et al. A reference consensus genetic map for molecular markers and economically important traits in faba bean (Vicia faba L.). BMC Genomics. 2013; 14:932 Available from: http://www.biomedcentral.com/1471-2164/14/932.

24. Trapnell C, Williams BA, Pertea G, Mortazavi A, Kwan G, van Baren MJ, et al. Transcript assembly and abundance estimation from RNA-Seq reveals thousands of new transcripts and switching among isoforms. Nat Biotechnol. 2011;28:511-5 Available from: http://www.pubmedcentral.nih. gov/articlerender.fcgi?artid=3146043\&tool=pmcentrez\&rendertype=abstract.

25. Burge SW, Daub J, Eberhardt R, Tate J, Barquist L, Nawrocki EP, et al. Rfam 11.0: 10 years of RNA families. Nucleic Acids Res. 2013;41:D226.

26. Sun L, Luo H, Bu D, Zhao G, Yu K, Zhang C, et al. Utilizing sequence intrinsic composition to classify protein-coding and long non-coding transcripts. Nucleic Acids Res. 2013;41(17):e166.

27. Trapnell C, Williams BA, Pertea G, Mortazavi A, Kwan G, Van Baren MJ, et al. Transcript assembly and quantification by RNA-Seq reveals unannotated transcripts and isoform switching during cell differentiation. Nat Biotechnol. 2010;28(5):511-5

28. Alex BM, Dhiral PP, Scott SA, Deepak M, Suzy MS, Ruchir RS, et al. Differentially expressed genes (DEGs) identified from RNA-Seq by DESeq and Cuffdiff compared to microarray. 2013;

29. Nawrocki EP, Kolbe DL, Eddy SR. Infernal 1.0: inference of RNA alignments. Bioinformatics. 2009;25:1335.

30. Fornara F, de Montaigu A, Coupland G. SnapShot: control of flowering in arabidopsis. Cell. 2010;141:3-5.

31. Lee J, Lee I. Regulation and function of SOC1, a flowering pathway integrator. J Exp Bot. 2010;61:2247-54

32. Hayama R, Sarid-Krebs L, Richter R, Fernández V, Jang S, Coupland G. PSEUDO RESPONSE REGULATORs stabilize CONSTANS protein to promote flowering in response to day length. EMBO J. 2017;36:904-18 Available from: http://emboj.embopress.org/lookup/doi/10.15252/embj. 201693907.

33. Tian H, Wang X, Guo H, Cheng Y, Hou C, Chen J-G, et al. NTL8 regulates trichome formation in Arabidopsis by directly activating R3 MYB genes TRY and TCL1. Plant Physiol. 2017;174:2363-75 Available from: http://www. plantphysiol.org/lookup/doi/10.1104/pp.17.00510.

34. Zhang D, Zheng Y. Molecular control of grass inflorescence development. Annu Rev Plant Biol. 2014;65:553.

35. Wang JW, Czech B, Weigel D. miR156-regulated SPL transcription factors define an endogenous flowering pathway in Arabidopsis thaliana. Cell. 2009;138:738-49.

36. Bouché F, Lobet $G$, Tocquin P, Périlleux C. FLOR-ID: an interactive database of flowering-time gene networks in Arabidopsis thaliana. Nucleic Acids Res. 2016;44:D1167-71

37. Kang C, Liu Z. Global identification and analysis of long non-coding RNAs in diploid strawberry Fragaria vesca during flower and fruit development. BMC Genomics. 2015:16:1-15.

38. Illumina. Understanding Illumina Quality Scores. Tech Note Inform. 2014;1-2. Available from: http://www.illumina.com/documents/products/technotes/ technote_understanding_quality_scores.pdf. 
39. Phred C. Scores Q. Quality Scores for Next-Generation Sequencing; 2000. p. $1-2$.

40. Feng N, Song G, Guan J, Chen K, Jia M, Huang D, et al. Transcriptome profiling of wheat inflorescence development from spikelet initiation to floral patterning identified stage-specific regulatory genes. Plant Physiol. 2017;174:1779-94 Available from: http://www.plantphysiol.org/lookup/doi/ 10.1104/pp.17.00310

41. Yan J, Chia J-C, Sheng H, Jung H, Zavodna T-O, Lu Z, et al. Arabidopsis pollen fertility requires the transcription factors CIT1 and SPL7 that regulate copper delivery to anthers and jasmonic acid synthesis. Plant Cell. 2017:tpc. 00363.2017. Available from: http:/www.plantcell.org/lookup/doi/10.1105/ tpc.17.00363.

42. Ling LZ, Zhang SD. Exploring the evolutionary differences of SBP-box genes targeted by miR156 and miR529 in plants. Genetica. 2012;140:317-24.

43. Jung JH, Lee HJ, Ryu JY, Park CM. SPL3/4/5 integrate developmental aging and photoperiodic signals into the FT-FD module in Arabidopsis flowering. Mol Plant. 2016;9:1647-59 Available from: https://doi.org/10.1016/j.molp. 2016.10 .014

44. Yamasaki H, Hayashi M, Fukazawa M, Kobayashi Y, Shikanai T. SQUAMOSA promoter binding protein-like7 is a central regulator for copper homeostasis in Arabidopsis. Plant Cell. 2009;21:347-61 Available from: http://www.plantcell.org/cgi/doi/10.1105/tpc.108.060137.

45. Carmona MJ, Calonje M, Martínez-Zapater JM. The FT/TFL1 gene family in grapevine. Plant Mol Biol. 2007;63:637-50.

46. Wang H, Pan J, Li Y, Lou D, Hu Y, Yu D. The DELLA-CONSTANS transcription factor cascade integrates gibberellic acid and photoperiod signaling to regulate flowering. Plant Physiol. 2016;172:479-88.

47. Niu Y, Figueroa P, Browse J. Characterization of JAZ-interacting bHLH transcription factors that regulate jasmonate responses in Arabidopsis. J Exp Bot. 2011;62:2143-54.

48. Schmid M, Davison TS, Henz SR, Pape UJ, Demar M, Vingron M, et al. A gene expression map of Arabidopsis thaliana development. Nat Genet. 2005:37:501-6

49. Yu S, Galvao VC, Zhang Y-C, Horrer D, Zhang T-Q, Hao Y-H, et al. Gibberellin regulates the Arabidopsis floral transition through miR156-targeted SQUAMOSA promoter binding-like transcription factors. Plant Cell. 2012;24: 3320-32 Available from: http://www.plantcell.org/cgi/doi/10.1105/tpc.112. 101014

50. Li C, Lu S. Molecular characterization of the SPL gene family in Populus trichocarpa. Available from: http://xueshu.baidu.com/s?wd=paperuri: \%2863dba9100800d2507dff44b971b28877\%29\&filter=sc_long_sign\&tn=SE_ xueshusource_2kduw22v\&sc_vurl=http://europepmc.org/articles/ PMC4035897/\&ie=utf-8\&sc_us=16354895411100889434.

51. Zhang X, An L, Nguyen TH, Liang H, Wang R, Liu X, et al. The cloning and functional characterization of peach CONSTANS and FLOWERING LOCUS T homologous genes PpCO and PpFT. PLoS One. 2015;10:1-16.

52. Hisamoto $Y$, Kashiwagi $H$, Kobayashi M. Use of flowering gene FLOWERING LOCUS T (FT) homologs in the phylogenetic analysis of bambusoid and early diverging grasses. J Plant Res. 2008;121:451-61 Available from: http:// link.springer.com/10.1007/s10265-008-0181-9.

53. Yamaguchi A, Kobayashi Y, Goto K, Abe M, Araki T. TWIN SISTER of FT (TSF) acts as a floral pathway integrator redundantly with FT. Plant Cell Physiol. 2005;46:1175-89.

54. EGO M, da Silva EM, e Silva GFF, Valente GT, Barrera Rojas CH, Vincentz M, et al. Functional and evolutionary analyses of the miR156 and miR529 families in land plants. BMC Plant Biol. 2016;16:40 Available from: http:// bmcplantbiol.biomedcentral.com/articles/10.1186/s12870-016-0716-5.

55. Zhang SD, Ling LZ, Zhang QF, Xu JD, Cheng L. Evolutionary comparison of two combinatorial regulators of SBP-box genes, MiR156 and MiR529, in plants. PLoS One. 2015;10:1-12.

56. Zhang D-D, Wang W-T, Xiong J, Xie X-M, Cui S-S, Zhao Z-G, et al. Long noncoding RNA LINC00305 promotes inflammation by activating the AHRRNF-KB pathway in human monocytes. Sci Rep. 2017;7:46204 Available from: http://www.nature.com/articles/srep46204

\section{Ready to submit your research? Choose BMC and benefit from:}

- fast, convenient online submission

- thorough peer review by experienced researchers in your field

- rapid publication on acceptance

- support for research data, including large and complex data types

- gold Open Access which fosters wider collaboration and increased citations

- maximum visibility for your research: over $100 \mathrm{M}$ website views per year

At BMC, research is always in progress.

Learn more biomedcentral.com/submissions 